

Original Research Article

http://dx.doi.org/10.20546/ijcmas.2016.506.036

\title{
Effect of Organic Acids and Probiotics on the Pond Ecosystem in the Culture Ponds of Litopenaeus vannamei
}

\author{
Gopal Anand Kandikatla* and Ramesh Babu Kondamudi \\ Department of Marine Living Resources and Research Centre, \\ Andhra University College of Science and Technology, Visakhapatnam, A.P, India \\ *Corresponding author
}

\section{Introduction}

The study was conducted at Vaadachepurupalli, Visakhapatnam district in Andhra Pradesh. Organic acids act as growth promoters and enhance the antimicrobial activity of the organism; they also enhance the nutrient digestibility, survival and maintain a static endogenous micro floral quantity and composition. As organic acid is very essential and useful it's called "A gut environment modifier (GEM) designed to improve feed quality and an alternative to antibiotics".
It improves digestibility of the aquaculture feeds, increases the feed intake of aquatic species, has a strong antimicrobial effect and acts against gram negative bacteria leads to prevention of the diseases, it acts as a feed hygiene and feed quality regulator (antimould), it reduces buffering capacity of the feed, It helps in the decrease of $\mathrm{pH}$ in the feed which prevents the ammonia formation in the faecal matter, It also helps in the faster acidification of stomach content towards optimal $\mathrm{pH}$ for pepsin digestion, acidification 
of the hepatopancreas, gut acidification, stimulation of enzyme secretion, improves protein digestion and also increases amino acid digestibility. In rearing ponds of Litopenaeus vannamei, the use of commercial probiotics has shown beneficial effects by improving survival, feed conversion, growth rate and keeping the parameters of water quality at optimum levels (Shariff et al., 2001; Wang et al., 2005). Studies of organic acids and probiotics to improve growth or survival in crustacean larvae are very scanty. The application of probiotics in the aquaculture ecosystems and in the feeds to the animal is one of the most promising areas where sustainable culture can be established and the practice of application of probiotics is reported by many aqua-culturists. Knowledge of probiotics has increased, currently it is know that these microorganisms have an antimicrobial effect through modifying the intestinal microbiota, secreting antibacterial substances like bacteriocins and organic acids (Myers., 2007). Organic acids and their salts are generally regarded as safe compounds and those with one or more carboxyl group ($\mathrm{COOH})$ in their structure are often used as antimicrobials in the livestock feed industry. In shrimp culture there are different bacterial strains used as probiotics and the popular probiotic bacteria belong to Nitrosomonas spp., Cellulomonas spp., Bacillus spp., Pediococcus spp., Nitrobacter spp., Rhodococus spp., Rhodobacter spp., Enterobacter spp., Lactobacillus spp., Actinomycetes spp., Pseudomonas spp., Saccharomyces spp., Denitrifying bacteria, Bifidobacterium, Carnobacterium, Alteronomonas spp., Streptococcus spp.,

\section{Materials and Methods}

The application of probiotics in the experimental ponds was followed uniformly in all the farms. The probiotic used for present study is SUPER BIOTIC (Plate 5 Fig B) which is a composition of water probiotics having the strength of 10 million colony forming units (CFU) i.e. $109 \mathrm{cfu} / \mathrm{g}$ of the probiotic was used. The probiotic strains in this SUPER BIOTIC were Bacillus spp. like Bacillus subtilis, Bacillus licheniformis, Bacillus megatirium and Bacillus polymixa. The probiotic was applied at the rate of $2 \mathrm{kgha}-$ 1 in the experimental pond A and 3kgha- 1 in the experimental pond $\mathrm{B}$ at the frequency of every 15 days during the three years of the study starting from 45 DOC (Table 1(b)). The probiotics application followed the same procedure in all the experimental ponds during the study period. The application of probiotics were followed by soaking the probiotics material in 4 liters of water overnight for leaching in non-contaminated fresh water and later applied uniformly all over the ponds. For the studies on the role of organic acids, the commercial organic acid used in the present study is BAYERS' BAYMIX LATIBON (Plate 5, Fig. A) which is composed of formic, lactic, benzoic and propionic acids. The organic acid BAYMIX LATIBON is applied at the rate of $3 \mathrm{gkg}-1$ feed in the experimental pond A and 5 gkg-1 feed (Table 1(a)) in the experimental pond $\mathrm{B}$ in both summer and winter crops during the study period starting from 30 DOC based on the requirement. Application of organic acids was stopped two days prior to the application of SUPER BIOTIC and was started two days after the application of SUPER BIOTIC. The Organic acid is mixed in the feed with commercially available feed binder "Gell it" which was applied $10 \mathrm{ml} / \mathrm{kg}$ feed. In the process of top dressing in the feed the organic acid were weighed with simple balance of sensitivity 10 gm and mixed thoroughly with the binder "Gell It". The feed was broadcasted in the ponds after drying of feed pellets for 20 to 30 minutes in shade. The feed for the experimental study ponds for every feeding time was freshly mixed with organic acid. Later the feed was dried in the shade and 
broadcasted in every feeding time. The application of the organic acid was followed every feeding time. The application of the organic acid was followed every day starting from 45 DOC during the culture in the entire crop period of both season's summer and winter in the three years of study period 2011 to 2013 .

\section{Estimation of Bacterial Load of Water}

Water samples from the selected culture ponds were collected in sterile glass bottles and brought to the laboratory in cold condition and bacterial loads were estimated within one hour of collection, by employing standard pour plate method. The samples were prepared by serial dilution method. One $\mathrm{ml}$ of diluted water sample was taken asceptically into sterile, dry petridish with the help of a pipette. The nutrient agar medium (Himedia, Bombay) in lukeworm state was poured onto the sample contained in the petridish, and then petridish was rotated gently in both clock and anticlockwise directions for uniform distribution of sample 41 solutions, triplicate sets were maintained for each direction. The petridishes were inverted after medium got solidified.

\section{Estimation of Bacterial Load of Shrimp}

Four shrimps collected from selected ponds at fortnightly interval for the estimation of bacteria loads, the standard methods were followed. The bacterial loads were estimated from gut, Hepatopancreas and Haemolymph. The weighed tissue sample approximately 60 to $80 \mathrm{mg}$ was taken aseptically from freshly sacrificed specimen into a known volume of sterile and cooled peptone water (Himedia, Bombay). The tissues were homogenized with the help of sterile glass rod in ice cold conditions. One $\mathrm{ml}$ of homogenate was taken into dry, sterile petridish aseptically with a pipette. The bacterial load was estimated as reported earlier and expressed in $\mathrm{CFU} / \mathrm{g}$. In case of water, the volume of water sample was collected from various locations of the ponds during different times of the day. The bacterial loads estimated and expressed in $\mathrm{CFU} / \mathrm{ml}$ Count was expressed in CFU/g.

Average number of colonies $=$

No. of CFU/g of the gut X Dilution factor Weight of gut sample

\section{Statistical Applications}

The statistical package used for interpreting the available data was GRAPHPAD PRISM 6.0 Scientific Software for evaluation of the total Vibrio colony (TVC) counts. Histograms were used to interpret the growth data of the results obtained. The pie charts were used to represent the total percentage production of shrimp Litopenaeus vannamei in Andhra Pradesh during the year 2011 and 2013. The pie charts were applied for representing the data of the survival rate and growth during the study period in all the ponds of the eight work stations. The results of the immunological indices were tabulated and represented by using the statistical tool 2-D line charts.

\section{Results and Discussion}

At Vadacheepurupalli during the culture periods in the summer crop and winter crop from the year 2011 to 2013, the parameters of salinity, $\mathrm{pH}$, and temperature in the study ponds were closely monitored and it was observed that the $\mathrm{pH}$ which was well maintained with the application of Organic acids and probiotics (). This control pond harvested at $19.0 \mathrm{~g}$ on $109^{\text {th }}$ day with the effect of Vibriosis spp..

The total Vibrio counts in the experimental pond $\mathrm{A}$ and pond $\mathrm{B}$ were recorded as $31.0 \times 10^{2}$ $\pm 0.25 \mathrm{cfu} \mathrm{ml}^{-1}$ and $3.10 \times 10^{2} \pm 0.23 \mathrm{cfu} \mathrm{ml}^{-1}$ in the pond water and $20.0 \times 10^{2} \pm 0.13 \mathrm{cfu} \mathrm{mg}^{-1}$ and $4.15 .12 \times 10^{2} \pm 0.37 \mathrm{cfu} \mathrm{mg}^{-1}$ in the shrimp 
gut at 125 days of culture respectively. The experimental ponds A and B were harvested normally at $27.5 \mathrm{~g}$ and $30.0 \mathrm{~g}$ on $120^{\text {th }}$ and $135^{\text {th }}$ day respectively.

Table 1 Total vibrio count of culture ponds at Vadacheepurupalli during summer in the year 2011

Control Pond

\begin{tabular}{|c|c|c|c|c|c|c|}
\hline S.No & $\begin{array}{c}\text { Days of } \\
\text { Culture }\end{array}$ & $\begin{array}{c}\text { Salinity } \\
(\mathrm{ppt})\end{array}$ & $\mathrm{Ph}$ & Temp $\left({ }^{\circ} \mathrm{C}\right)$ & $\begin{array}{c}\text { TVC water } \\
\text { cfuml }^{-1}\end{array}$ & $\begin{array}{c}\text { TVC gut } \\
\text { cfumg }^{-1}\end{array}$ \\
\hline 1 & $\begin{array}{c}\text { Before } \\
\text { stocking }\end{array}$ & 25 & 8.1 & 29 & $0.24 \times 10^{2} \pm 0.27$ & - \\
\hline 2 & 25 & 30 & 8.5 & 30 & $0.28 \times 10^{2} \pm 0.60$ & - \\
\hline 3 & 50 & 31 & 7.9 & 31 & $1.55 \times 10^{2} \pm 0.41$ & $1.23 \times 10^{2} \pm 0.34$ \\
\hline 4 & 75 & 35 & 8.3 & 32 & $30.0 \times 10^{2} \pm 0.47$ & $24.8 \times 10^{2} \pm 0.41$ \\
\hline 5 & 100 & 39 & 8.5 & 32 & $32.7 \times 10^{2} \pm 0.81$ & $25.7 \times 10^{2} \pm 0.63$ \\
\hline 6 & 125 & - & - & - & - & - \\
\hline \multicolumn{7}{c}{ Control Pond harvested due to Vibriosis at $19 \mathrm{~g}$ on $109^{\text {th }}$ day } \\
\hline
\end{tabular}

Experimental Pond A

\begin{tabular}{|c|c|c|l|c|c|c|}
\hline S.No & $\begin{array}{c}\text { Days of } \\
\text { Culture }\end{array}$ & $\begin{array}{c}\text { Salinity } \\
(\mathrm{ppt})\end{array}$ & $\mathrm{pH}$ & $\mathrm{Temp}\left({ }^{\circ} \mathrm{C}\right)$ & $\begin{array}{c}\mathrm{TVC}^{-1} \text { water } \\
\text { cfuml }^{-1}\end{array}$ & $\begin{array}{c}\mathrm{TVC} \mathrm{gut}^{-1} \\
\text { cfumg }^{-1}\end{array}$ \\
\hline 1 & $\begin{array}{c}\text { Before } \\
\text { stocking }\end{array}$ & 26 & 7.8 & 29 & - & - \\
\hline 2 & 25 & 33 & 8.2 & 29 & - & - \\
\hline 3 & 50 & 35 & 8.5 & 30 & $1.48 \times 10^{2} \pm 0.15$ & $1.63 \times 10^{2} \pm 0.36$ \\
\hline 4 & 75 & 36 & 8.3 & 31 & $2.63 \times 10^{2} \pm 0.23$ & $2.28 \times 10^{2} \pm 0.15$ \\
\hline 5 & 100 & 37 & 8.7 & 32 & $28.0 \times 10^{2} \pm 0.25$ & $19.7 \times 10^{2} \pm 0.29$ \\
\hline 6 & 125 & 36 & 8.6 & 32 & $31.0 \times 10^{2} \pm 0.25$ & $20.0 \times 10^{2} \pm 0.13$ \\
\hline
\end{tabular}

Pond A harvested normally at $27.5 \mathrm{~g}$ on $130^{\text {th }}$ day

Experimental Pond B

\begin{tabular}{|c|c|c|c|c|c|c|}
\hline S.No & $\begin{array}{c}\text { Days of } \\
\text { Culture }\end{array}$ & $\begin{array}{c}\text { Salinity } \\
(\mathrm{ppt})\end{array}$ & $\mathrm{pH}$ & Temp $\left({ }^{\circ} \mathrm{C}\right)$ & $\begin{array}{c}\mathrm{TVC}^{\mathrm{N}} \text { water } \\
\text { cfuml }^{-1}\end{array}$ & $\begin{array}{c}\text { TVC gut } \\
\text { cfumg }^{-1}\end{array}$ \\
\hline 1 & $\begin{array}{l}\text { Before } \\
\text { stocking }\end{array}$ & 26 & 8.2 & 28 & - & - \\
\hline 2 & 25 & 34 & 8.2 & 29 & - & - \\
\hline 3 & 50 & 35 & 8.5 & 31 & $0.25 \times 10^{2} \pm 0.36$ & $0.23 \times 10^{2} \pm 0.35$ \\
\hline 4 & 75 & 36 & 8.4 & 31 & $1.63 \times 10^{2} \pm 0.46$ & $1.83 \times 10^{2} \pm 0.16$ \\
\hline 5 & 100 & 37 & 8.7 & 32 & $2.63 \times 10^{2} \pm 0.32$ & $3.32 \times 10^{2} \pm 0.23$ \\
\hline 6 & 125 & 39 & 8.4 & 33 & $3.10 \times 10^{2} \pm 0.23$ & $4.15 \times 10^{2} \pm 0.37$ \\
\hline
\end{tabular}

Pond B harvested normally at $30 \mathrm{~g}$ on $135^{\text {th }}$ day 
Table 2 Total vibrio count of culture ponds at Vadacheepurupalli during winter in the year 2011

Control Pond

\begin{tabular}{|c|c|c|c|c|c|c|}
\hline S.No & $\begin{array}{l}\text { Days of } \\
\text { Culture }\end{array}$ & $\begin{array}{c}\text { Salinity } \\
(\mathrm{ppt})\end{array}$ & $\mathrm{pH}$ & Temp $\left({ }^{\circ} \mathrm{C}\right)$ & $\begin{array}{c}\text { TVC water } \\
\text { cfuml }^{-1}\end{array}$ & $\begin{array}{c}\text { TVC gut } \\
\text { cfumg }^{-1}\end{array}$ \\
\hline 1 & $\begin{array}{l}\text { Before } \\
\text { stocking }\end{array}$ & 23 & 8.2 & 28 & - & - \\
\hline 2 & 25 & 25 & 8.6 & 29 & $0.28 \times 10^{2} \pm 0.60$ & $0.15 \times 10^{2} \pm 0.13$ \\
\hline 3 & 50 & - & - & - & - & - \\
\hline 4 & 75 & - & - & - & - & - \\
\hline 5 & 100 & - & - & - & - & - \\
\hline 6 & 125 & - & - & - & - & - \\
\hline
\end{tabular}

Control Pond harvested due to Vibriosis at $3.2 \mathrm{~g}$ on $30^{\text {th }}$ day

Experimental Pond A

\begin{tabular}{|l|l|c|l|c|c|c|}
\hline S.No & $\begin{array}{l}\text { Days of } \\
\text { Culture }\end{array}$ & $\begin{array}{c}\text { Salinity } \\
(\mathrm{ppt})\end{array}$ & $\mathrm{pH}$ & Temp $\left({ }^{\circ} \mathrm{C}\right)$ & $\begin{array}{c}\mathrm{TVC}^{2} \text { water } \\
\text { cfuml }^{-1}\end{array}$ & $\begin{array}{c}\text { TVC gut } \\
\text { cfumg }^{-1}\end{array}$ \\
\hline 1 & $\begin{array}{l}\text { Before } \\
\text { ttocking }\end{array}$ & 22 & 7.8 & 28 & - & - \\
\hline 2 & 25 & 24 & 8.1 & 27 & $0.11 \times 10^{2} \pm 0.10$ & - \\
\hline 3 & 50 & 25 & 8.2 & 27 & $0.31 \times 10^{2} \pm 0.52$ & $0.27 \times 10^{2} \pm 0.29$ \\
\hline 4 & 75 & 24 & 7.9 & 25 & $1.78 \times 10^{2} \pm 0.38$ & $2.45 \times 10^{2} \pm 0.27$ \\
\hline 5 & 100 & 25 & 8.4 & 25 & $2.33 \times 10^{2} \pm 0.30$ & $2.77 \times 10^{2} \pm 0.31$ \\
\hline 6 & 125 & 21 & 8.3 & 26 & $3.92 \times 10^{2} \pm 0.55$ & $3.50 \times 10^{2} \pm 0.23$ \\
\hline
\end{tabular}

Experimental Pond B

\begin{tabular}{|c|c|c|l|c|c|c|}
\hline S.No & $\begin{array}{c}\text { Days of } \\
\text { Culture }\end{array}$ & $\begin{array}{c}\text { Salinity } \\
(\mathrm{ppt})\end{array}$ & $\mathrm{pH}$ & Temp $\left({ }^{\circ} \mathrm{C}\right)$ & $\begin{array}{c}\text { TVC water } \\
\text { cfuml }^{-1}\end{array}$ & $\begin{array}{c}\mathrm{TVC}_{\text {gut }} \\
\text { cfumg }^{-1}\end{array}$ \\
\hline 1 & $\begin{array}{c}\text { Before } \\
\text { stocking }\end{array}$ & 16 & 7.5 & 28 & - & - \\
\hline 2 & 25 & 17 & 7.9 & 27 & - & - \\
\hline 3 & 50 & 19 & 8.4 & 26 & $0.17 \times 10^{2} \pm 0.15$ & - \\
\hline 4 & 75 & 19 & 8.5 & 26 & $1.03 \times 10^{2} \pm 0.45$ & $1.00 \times 10^{2} \pm 0.12$ \\
\hline 5 & 100 & 17 & 8.2 & 25 & $1.75 \times 10^{2} \pm 0.16$ & $1.30 \times 10^{2} \pm 0.16$ \\
\hline 6 & 125 & 20 & 8.1 & 25 & $2.08 \times 10^{2} \pm 0.13$ & $2.04 \times 10^{2} \pm 0.19$ \\
\hline
\end{tabular}


Table 3 Total vibrio count of culture ponds at Vadacheepurupalli during summer in the year 2012

Control Pond

\begin{tabular}{|c|c|c|c|c|c|c|}
\hline S.No & $\begin{array}{l}\text { Days of } \\
\text { Culture }\end{array}$ & $\begin{array}{c}\text { Salinity } \\
(\mathrm{ppt})\end{array}$ & $\mathrm{pH}$ & Temp $\left({ }^{\circ} \mathrm{C}\right)$ & $\begin{array}{c}\text { TVC water } \\
\text { cfuml }^{-1}\end{array}$ & $\begin{array}{c}\text { TVC gut } \\
\text { cfumg }^{-1}\end{array}$ \\
\hline 1 & $\begin{array}{c}\text { Before } \\
\text { stocking }\end{array}$ & 25 & 8.2 & 30 & $0.22 \times 10^{2} \pm 0.27$ & - \\
\hline 2 & 25 & 30 & 8.9 & 31 & $0.30 \times 10^{2} \pm 0.60$ & - \\
\hline 3 & 50 & 35 & 7.8 & 33 & $1.57 \times 10^{2} \pm 0.40$ & $1.57 \times 10^{2} \pm 0.40$ \\
\hline 4 & 75 & 39 & 8.5 & 33 & $30.0 \times 10^{2} \pm 0.47$ & $0.49 \times 10^{2} \pm 0.24$ \\
\hline 5 & 100 & 25 & 8.2 & 30 & $37.25 \times 10^{2} \pm 0.23$ & $33.10 \times 10^{2} \pm 0.47$ \\
\hline 6 & 125 & 30 & 8.9 & 31 & $40.21 \times 10^{2} \pm 0.13$ & $39.23 \times 10^{2} \pm 0.85$ \\
\hline
\end{tabular}

Control Pond harvested normally at $26 \mathrm{~g}$ on $127^{\text {th }}$ day

Experimental Pond A

\begin{tabular}{|c|c|c|c|c|c|c|}
\hline S.No & $\begin{array}{c}\text { Days of } \\
\text { Culture }\end{array}$ & $\begin{array}{c}\text { Salinity } \\
(\mathrm{ppt})\end{array}$ & $\mathrm{pH}$ & Temp $\left({ }^{\circ} \mathrm{C}\right)$ & $\begin{array}{c}\text { TVC water } \\
\text { cfuml }^{-1}\end{array}$ & $\begin{array}{c}\text { TVC gut } \\
\text { cfumg }^{-1}\end{array}$ \\
\hline 1 & $\begin{array}{c}\text { Before } \\
\text { stocking }\end{array}$ & 26 & 7.8 & 28 & - & - \\
\hline 2 & 25 & 27 & 8.5 & 30 & - & - \\
\hline 3 & 50 & 32 & 8.5 & 32 & - & - \\
\hline 4 & 75 & 35 & 7.9 & 33 & $0.22 \times 10^{2} \pm 0.47$ & $0.17 \times 10^{2} \pm 0.22$ \\
\hline 5 & 100 & 36 & 8.6 & 32 & $2.25 \times 10^{2} \pm 0.16$ & $3.97 \times 10^{2} \pm 0.42$ \\
\hline 6 & 125 & 32 & 8.2 & 32 & $3.25 \times 10^{2} \pm 0.60$ & $4.25 \times 10^{2} \pm 0.13$ \\
\hline
\end{tabular}

Pond A harvested normally at $30 \mathrm{~g}$ on $132^{\text {nd }}$ day

\section{Experimental Pond B}

\begin{tabular}{|c|c|c|c|c|c|c|}
\hline S.No & $\begin{array}{l}\text { Days of } \\
\text { Culture }\end{array}$ & $\begin{array}{c}\text { Salinity } \\
(\mathrm{ppt})\end{array}$ & $\mathrm{pH}$ & Temp $\left({ }^{\circ} \mathrm{C}\right)$ & $\begin{array}{c}\text { TVC water } \\
\text { cfuml }^{-1}\end{array}$ & $\begin{array}{c}\text { TVC gut } \\
\text { cfumg }^{-1}\end{array}$ \\
\hline 1 & $\begin{array}{l}\text { Before } \\
\text { stocking }\end{array}$ & 25 & 7.9 & 28 & - & - \\
\hline 2 & 25 & 28 & 8 & 30 & - & - \\
\hline 3 & 50 & 32 & 8.6 & 32 & - & - \\
\hline 4 & 75 & 35 & 8.6 & 33 & $0.26 \times 10^{2} \pm 0.47$ & - \\
\hline 5 & 100 & 37 & 8.7 & 32 & $0.41 \times 10^{2} \pm 0.49$ & $0.26 \times 10^{2} \pm 0.20$ \\
\hline 6 & 125 & 35 & 8.5 & 31 & $0.64 \times 10^{2} \pm 0.61$ & $0.33 \times 10^{2} \pm 0.54$ \\
\hline
\end{tabular}


Table 4 Total vibrio count of culture ponds at Vadacheepurupalli during winter in the year 2012

\section{Control Pond}

\begin{tabular}{|c|c|c|c|c|c|c|}
\hline S.No & $\begin{array}{l}\text { Days of } \\
\text { Culture }\end{array}$ & $\begin{array}{c}\text { Salinity } \\
(\mathrm{ppt})\end{array}$ & $\mathrm{pH}$ & Temp $\left({ }^{\circ} \mathrm{C}\right)$ & $\begin{array}{c}\text { TVC water } \\
\text { cfuml }^{-1}\end{array}$ & $\begin{array}{c}\text { TVC gut } \\
\text { cfumg }^{-1}\end{array}$ \\
\hline 1 & $\begin{array}{l}\text { Before } \\
\text { stocking }\end{array}$ & 14 & 8.5 & 28 & - & - \\
\hline 2 & 25 & 17 & 7.9 & 29 & $8.97 \times 10^{2} \pm 0.38$ & $2.65 \times 10^{2} \pm 0.19$ \\
\hline 3 & 50 & 18 & 8.8 & 27 & $5.62 \times 10^{2} \pm 0.25$ & $5.13 \times 10^{2} \pm 0.33$ \\
\hline 4 & 75 & 17 & 8.7 & 26 & $9.79 \times 10^{2} \pm 0.38$ & $7.22 \times 10^{2} \pm 0.19$ \\
\hline 5 & 100 & 17 & 8.5 & 26 & $26.5 \times 10^{2} \pm 0.19$ & $37.8 \times 10^{2} \pm 0.46$ \\
\hline 6 & 125 & 18 & 8.8 & 27 & $29.0 \times 10^{2} \pm 0.21$ & $40.10 \times 10^{2} \pm 0.17$ \\
\hline
\end{tabular}

Control Pond harvested normally at $22 \mathrm{~g}$ on $130^{\text {th }}$ day

\section{Experimental Pond A}

\begin{tabular}{|c|c|c|c|c|c|c|}
\hline S.No & $\begin{array}{l}\text { Days of } \\
\text { Culture }\end{array}$ & $\begin{array}{c}\text { Salinity } \\
(\mathrm{ppt})\end{array}$ & $\mathrm{pH}$ & Temp $\left({ }^{\circ} \mathrm{C}\right)$ & $\begin{array}{c}\text { TVC water } \\
\text { cfuml }^{-1}\end{array}$ & $\begin{array}{c}\text { TVC gut } \\
\text { cfumg }^{-1}\end{array}$ \\
\hline 1 & $\begin{array}{c}\text { Before } \\
\text { stocking }\end{array}$ & 15 & 8.1 & 28 & - & - \\
\hline 2 & 25 & 15 & 7.9 & 29 & $0.14 \times 10^{2} \pm 0.22$ & - \\
\hline 3 & 50 & 17 & 8.8 & 27 & $0.20 \times 10^{2} \pm 0.38$ & $0.16 \times 10^{2} \pm 0.20$ \\
\hline 4 & 75 & 17 & 8.7 & 26 & $3.17 \times 10^{2} \pm 0.62$ & $2.62 \times 10^{2} \pm 0.67$ \\
\hline 5 & 100 & 16 & 8.5 & 26 & $4.30 \times 10^{2} \pm 0.21$ & $3.50 \times 10^{2} \pm 0.54$ \\
\hline 6 & 125 & 17 & 8.6 & 27 & $5.10 \times 10^{2} \pm 0.23$ & $4.09 \times 10^{2} \pm 0.55$ \\
\hline
\end{tabular}

\section{Experimental Pond B}

\begin{tabular}{|c|c|c|c|c|c|c|}
\hline S.No & $\begin{array}{l}\text { Days of } \\
\text { Culture }\end{array}$ & $\begin{array}{c}\text { Salinity } \\
(\mathrm{ppt})\end{array}$ & $\mathrm{pH}$ & Temp $\left({ }^{\circ} \mathrm{C}\right)$ & $\begin{array}{c}\text { TVC water } \\
\text { cfuml }^{-1}\end{array}$ & $\begin{array}{c}\text { TVC gut } \\
\text { cfumg }^{-1}\end{array}$ \\
\hline 1 & $\begin{array}{c}\text { Before } \\
\text { stocking }\end{array}$ & 15 & 8.2 & 26 & - & - \\
\hline 2 & 25 & 16 & 8.1 & 27 & - & - \\
\hline 3 & 50 & 16 & 8.8 & 26 & - & - \\
\hline 4 & 75 & 17 & 8.7 & 26 & $0.12 \times 10^{2} \pm 0.14$ & $0.17 \times 10^{2} \pm 0.14$ \\
\hline 5 & 100 & 16 & 8.9 & 25 & $2.17 \times 10^{2} \pm 0.50$ & $2.48 \times 10^{2} \pm 0.35$ \\
\hline 6 & 125 & 17 & 8.7 & 25 & $3.30 \times 10^{2} \pm 0.15$ & $3.22 \times 10^{2} \pm 0.23$ \\
\hline
\end{tabular}


Table 5 Total vibrio count of culture ponds at Vadacheepurupalli during summer in the year 2013

\section{Control Pond}

\begin{tabular}{|c|c|c|c|c|c|c|}
\hline S.No & $\begin{array}{l}\text { Days of } \\
\text { Culture }\end{array}$ & $\begin{array}{c}\text { Salinity } \\
(\mathrm{ppt})\end{array}$ & $\mathrm{pH}$ & Temp $\left({ }^{\circ} \mathrm{C}\right)$ & $\begin{array}{c}\text { TVC water } \\
\text { cfuml }^{-1}\end{array}$ & $\begin{array}{c}\text { TVC gut } \\
\text { cfumg }^{-1}\end{array}$ \\
\hline 1 & $\begin{array}{c}\text { Before } \\
\text { thocking }\end{array}$ & 18 & 7.8 & 28 & - & - \\
\hline 2 & 25 & 20 & 8.7 & 30 & - & - \\
\hline 3 & 50 & 20 & 8.8 & 32 & $0.18 \times 10^{2} \pm 0.43$ & - \\
\hline 4 & 75 & 19 & 7.8 & 31 & $0.27 \times 10^{2} \pm 0.47$ & $0.50 \times 10^{2} \pm 1.03$ \\
\hline 5 & 100 & 19 & 8.5 & 30 & $4.10 \times 10^{2} \pm 0.84$ & $3.23 \times 10^{2} \pm 0.44$ \\
\hline 6 & 125 & 20 & 8.8 & 32 & $5.12 \times 10^{2} \pm 0.62$ & $4.56 \times 10^{2} \pm 0.65$ \\
\hline
\end{tabular}

Control Pond harvested normally at $22 \mathrm{~g}$ on $127^{\text {th }}$ day

Experimental Pond A

\begin{tabular}{|c|c|c|c|c|c|c|}
\hline No & $\begin{array}{l}\text { Days of } \\
\text { Culture }\end{array}$ & $\begin{array}{c}\text { Salinity } \\
(\mathrm{ppt})\end{array}$ & $\mathrm{pH}$ & Temp $\left({ }^{\circ} \mathrm{C}\right)$ & $\begin{array}{c}\text { TVC water } \\
\text { cfuml }^{-1}\end{array}$ & $\begin{array}{c}\text { TVC gut } \\
\text { cfumg }^{-1}\end{array}$ \\
\hline 1 & $\begin{array}{l}\text { Before } \\
\text { stocking }\end{array}$ & 19 & 8.2 & 29 & - & - \\
\hline 2 & 25 & 20 & 8.4 & 30 & $0.16 \times 10^{2} \pm 0.52$ & - \\
\hline 3 & 50 & 21 & 8.5 & 31 & $0.20 \times 10^{2} \pm 0.54$ & $0.17 \times 10^{2} \pm 0.21$ \\
\hline 4 & 75 & 20 & 8.4 & 29 & $0.22 \times 10^{2} \pm 0.23$ & $1.42 \times 10^{2} \pm 0.61$ \\
\hline 5 & 100 & 20 & 8.3 & 30 & $1.39 \times 10^{2} \pm 0.45$ & $2.86 \times 10^{2} \pm 0.85$ \\
\hline 6 & 125 & 21 & 8.5 & 31 & $28.7 \times 10^{2} \pm 0.63$ & $9.27 \times 10^{2} \pm 0.56$ \\
\hline
\end{tabular}

Pond A harvested normally at $31 \mathrm{~g}$ on $129^{\text {th }}$ day

Experimental Pond B

\begin{tabular}{|c|c|c|c|c|c|c|}
\hline S.No & $\begin{array}{l}\text { Days of } \\
\text { Culture }\end{array}$ & $\begin{array}{c}\text { Salinity } \\
(\mathrm{ppt})\end{array}$ & $\mathrm{pH}$ & Temp $\left({ }^{\circ} \mathrm{C}\right)$ & $\begin{array}{c}\text { TVC water } \\
\text { cfuml }^{-1}\end{array}$ & $\begin{array}{c}\text { TVC gut } \\
\text { cfumg }^{-1}\end{array}$ \\
\hline 1 & $\begin{array}{l}\text { Before } \\
\text { stocking }\end{array}$ & 18 & 8.1 & 28 & - & - \\
\hline 2 & 25 & 19 & 7.9 & 30 & $0.19 \times 10^{2} \pm 0.27$ & - \\
\hline 3 & 50 & 20 & 8.5 & 30 & $0.23 \times 10^{2} \pm 0.32$ & - \\
\hline 4 & 75 & 18 & 8.1 & 29 & $0.29 \times 10^{2} \pm 0.23$ & - \\
\hline 5 & 100 & 20 & 8.2 & 30 & $1.30 \times 10^{2} \pm 0.41$ & - \\
\hline 6 & 125 & 20 & 8.5 & 32 & $2.35 \times 10^{2} \pm 0.52$ & $0.83 \times 10^{2} \pm 0.49$ \\
\hline
\end{tabular}

Pond $\mathrm{B}$ harvested normally at $33.2 \mathrm{~g}$ on $129^{\text {th }}$ day 
Table 6 Total vibrio count of culture ponds at Vadacheepurupalli during winter in the year 2013

\section{Control Pond}

\begin{tabular}{|c|c|c|c|c|c|c|}
\hline S.No & $\begin{array}{l}\text { Days of } \\
\text { Culture }\end{array}$ & $\begin{array}{c}\text { Salinity } \\
(\mathrm{ppt})\end{array}$ & $\mathrm{pH}$ & Temp $\left({ }^{\circ} \mathrm{C}\right)$ & $\begin{array}{c}\text { TVC water } \\
\text { cfuml }^{-1}\end{array}$ & $\begin{array}{c}\text { TVC gut } \\
\text { cfumg }^{-1}\end{array}$ \\
\hline 1 & $\begin{array}{l}\text { Before } \\
\text { stocking }\end{array}$ & 16 & 7.1 & 29 & $0.72 \times 10^{2} \pm 0.56$ & - \\
\hline 2 & 25 & 19 & 7.3 & 28 & $2.28 \times 10^{2} \pm 0.60$ & $2.35 \times 10^{2} \pm 0.70$ \\
\hline 3 & 50 & - & - & - & - & - \\
\hline 4 & 75 & - & - & - & - & - \\
\hline 5 & 100 & - & - & - & - & - \\
\hline 6 & 125 & - & - & - & - & - \\
\hline
\end{tabular}

\section{Experimental Pond A}

\begin{tabular}{|c|c|c|c|c|c|c|}
\hline S.No & $\begin{array}{l}\text { Days of } \\
\text { Culture }\end{array}$ & $\begin{array}{c}\text { Salinity } \\
(\mathrm{ppt})\end{array}$ & $\mathrm{pH}$ & Temp $\left({ }^{\circ} \mathrm{C}\right)$ & $\begin{array}{c}\text { TVC water } \\
\text { cfuml }^{-1}\end{array}$ & $\begin{array}{c}\text { TVC gut } \\
\text { cfumg }^{-1}\end{array}$ \\
\hline 1 & $\begin{array}{l}\text { Before } \\
\text { stocking }\end{array}$ & 15 & 7.8 & 28 & $0.38 \times 10^{2} \pm 0.46$ & - \\
\hline 2 & 25 & 17 & 8.1 & 28 & $1.31 \times 10^{2} \pm 0.30$ & $1.28 \times 10^{2} \pm 0.63$ \\
\hline 3 & 50 & - & - & - & - & - \\
\hline 4 & 75 & - & - & - & - & - \\
\hline 5 & 100 & - & - & - & - & \\
\hline 6 & 125 & - & - & - & - & - \\
\hline
\end{tabular}

Pond A harvested due to White Spot Disease at $4 \mathrm{~g}$ on $25^{\text {th }}$ day

\section{Experimental Pond B}

\begin{tabular}{|c|c|c|c|c|c|c|}
\hline S.No & $\begin{array}{l}\text { Days of } \\
\text { Culture }\end{array}$ & $\begin{array}{c}\text { Salinity } \\
(\mathrm{ppt})\end{array}$ & $\mathrm{pH}$ & Temp $\left({ }^{\circ} \mathrm{C}\right)$ & $\begin{array}{c}\text { TVC water } \\
\text { cfuml }^{-1}\end{array}$ & $\begin{array}{c}\text { TVC gut } \\
\text { cfumg }^{-1}\end{array}$ \\
\hline 1 & $\begin{array}{l}\text { Before } \\
\text { Stocking }\end{array}$ & 16 & 7.5 & 28 & $0.29 \times 10^{2} \pm 0.60$ & - \\
\hline 2 & 25 & 17 & 8.3 & 27 & $1.52 \times 10^{2} \pm 0.45$ & $1.68 \times 10^{2} \pm 0.71$ \\
\hline 3 & 50 & - & - & - & - & - \\
\hline 4 & 75 & - & - & - & - & - \\
\hline 5 & 100 & - & - & - & - & - \\
\hline 6 & 125 & - & - & - & - & - \\
\hline
\end{tabular}

Pond B harvested due to White Spot Disease at $4 \mathrm{~g}$ on $25^{\text {th }}$ day 
Fig.1 Total vibrio count of culture ponds at Vadacheepurupalli during summer in the year 2011

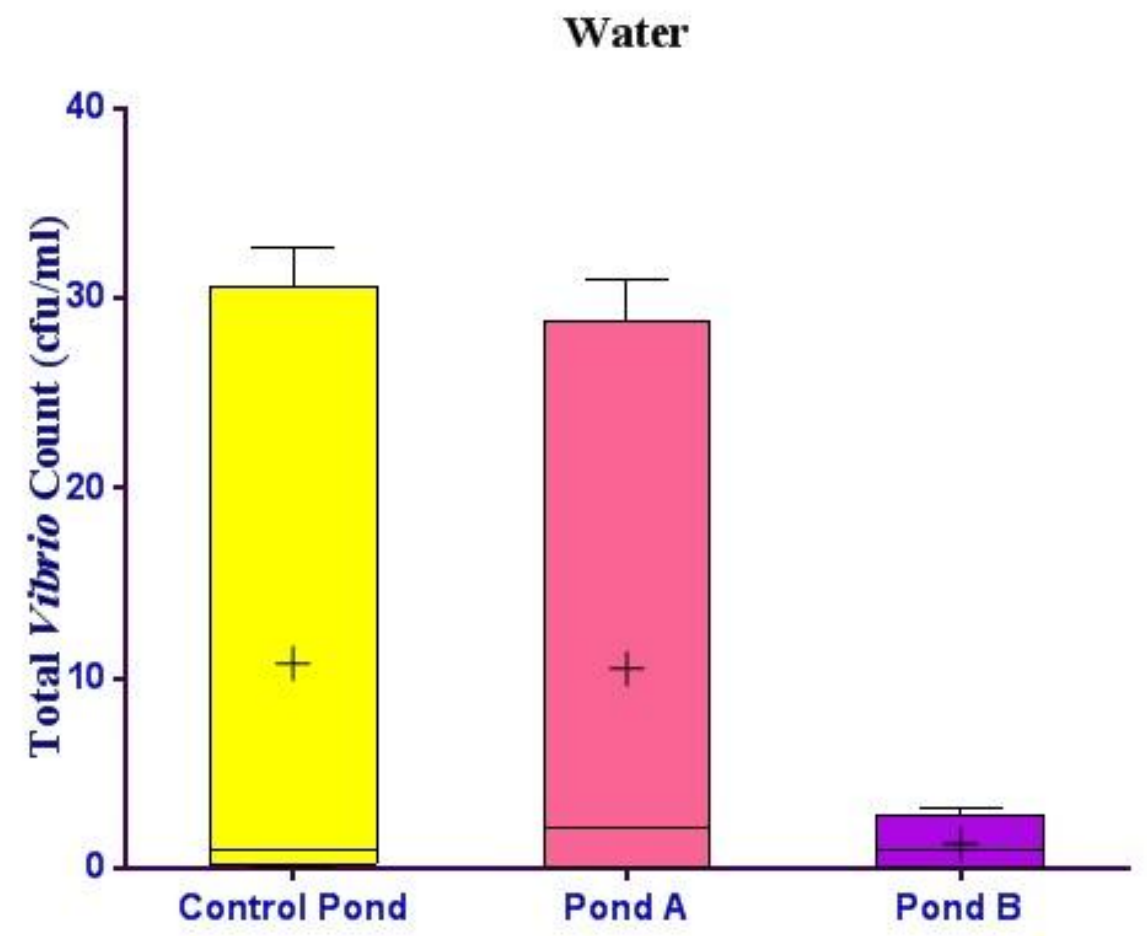

Gut

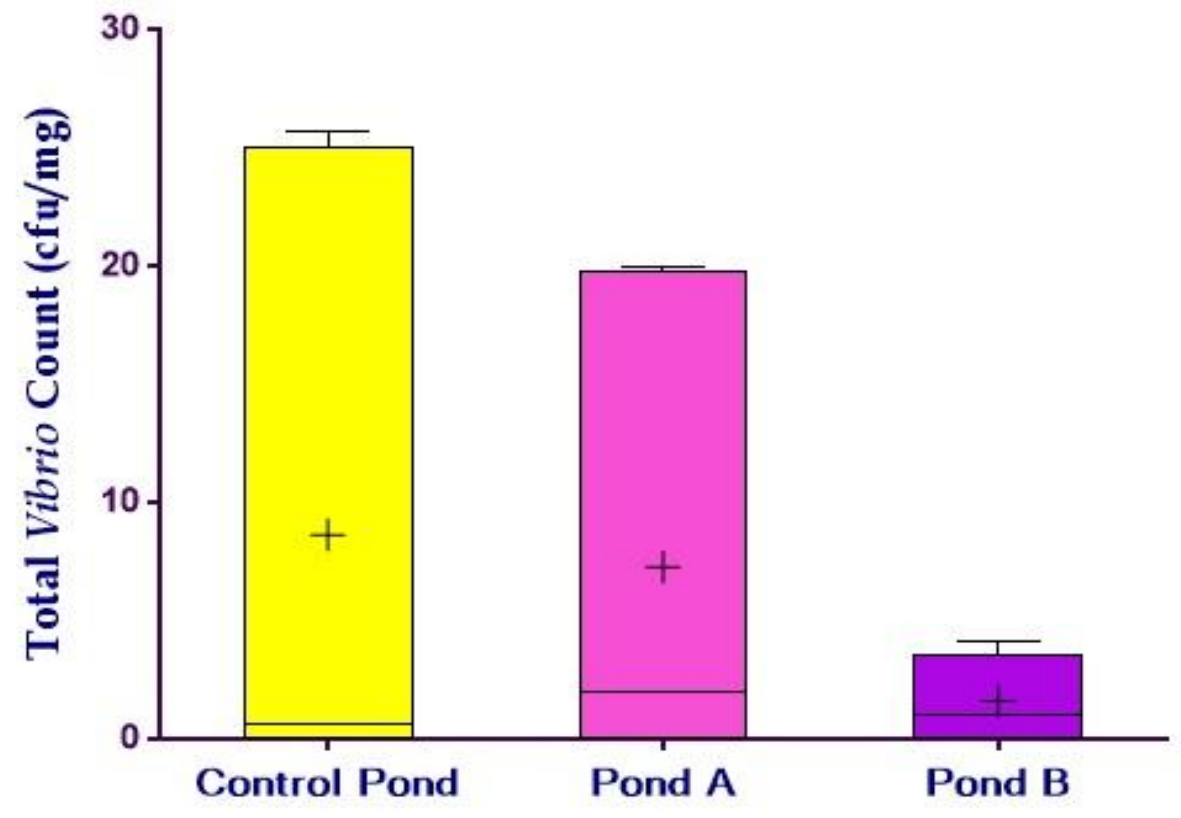


Fig.2 Total vibrio count of culture ponds at Vadacheepurupalli during winter in the year 2011

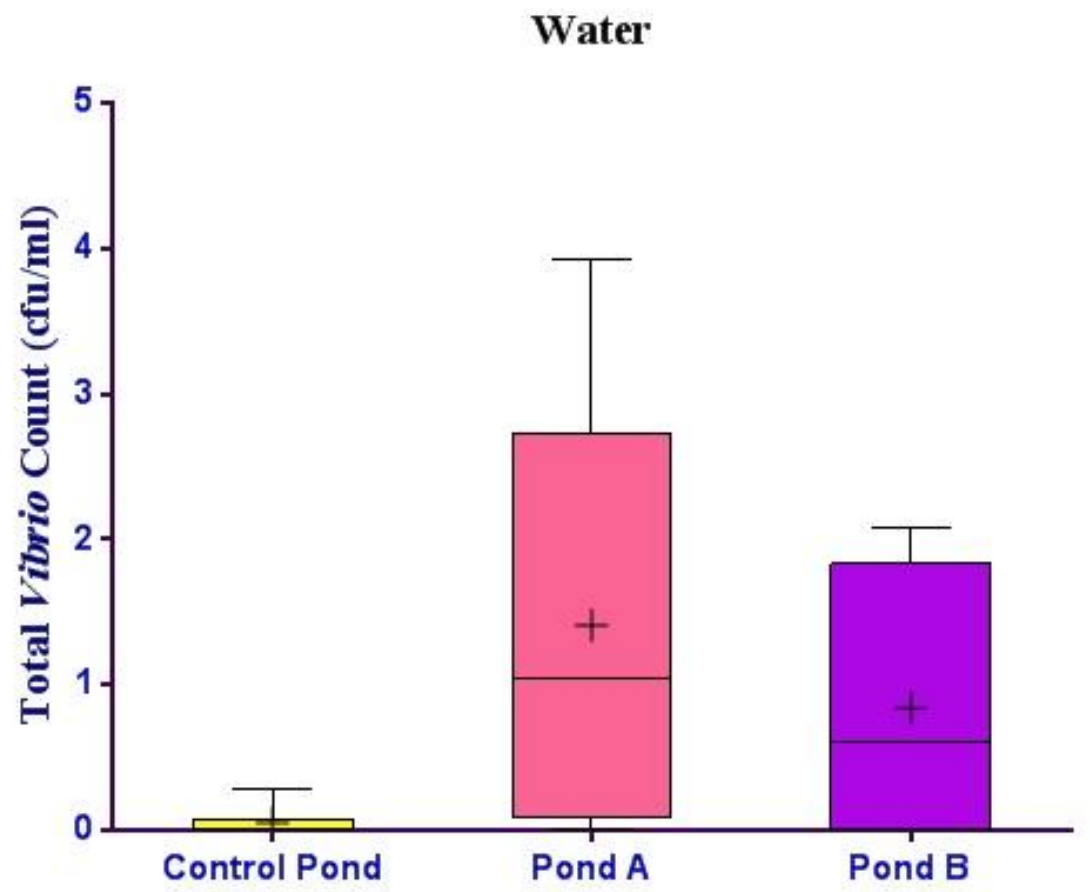

Gut

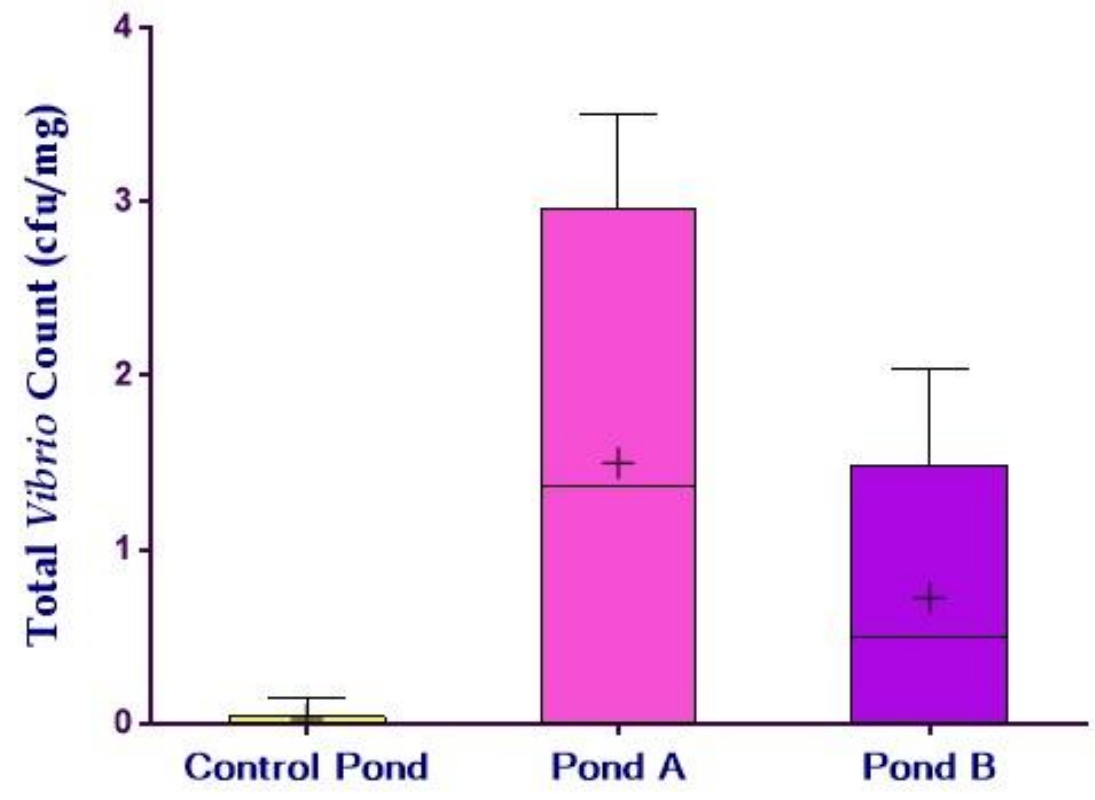


Fig.3 Total vibrio count of culture ponds at Vadacheepurupalli during summer in the year 2012

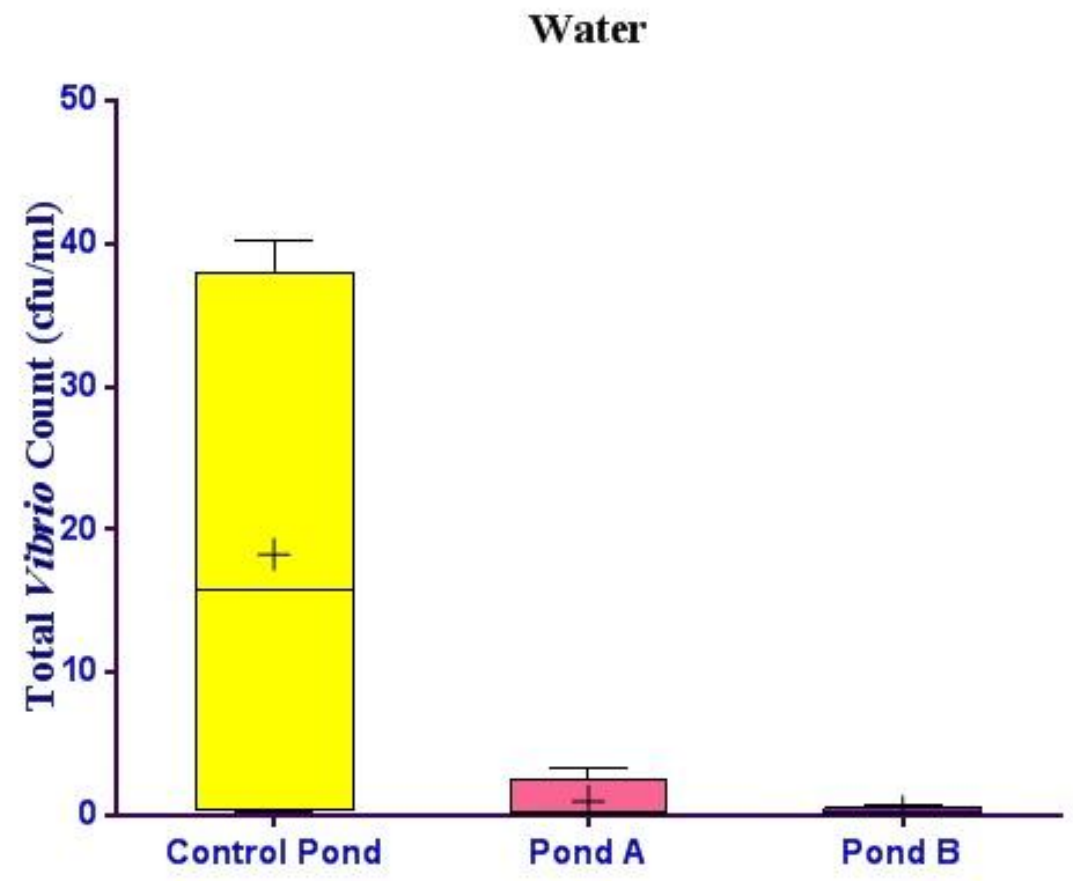

\section{Gut}

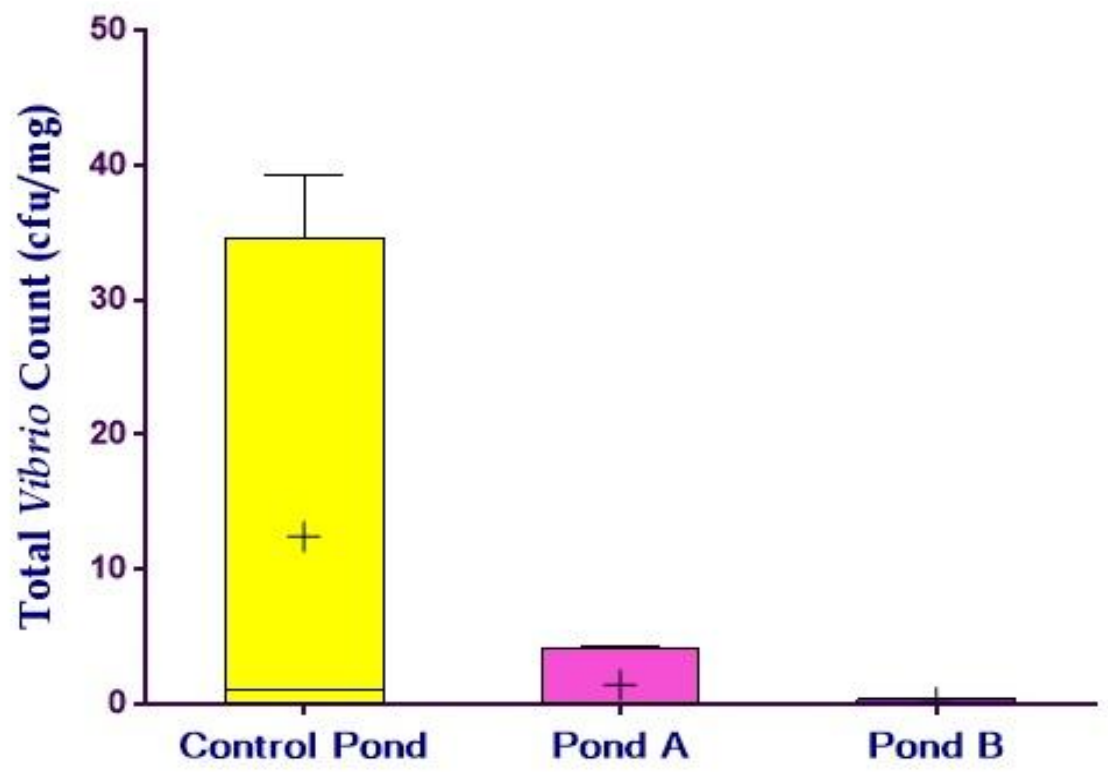


Fig.4 Total vibrio count of culture ponds at Vadacheepurupalli during winter in the year 2012

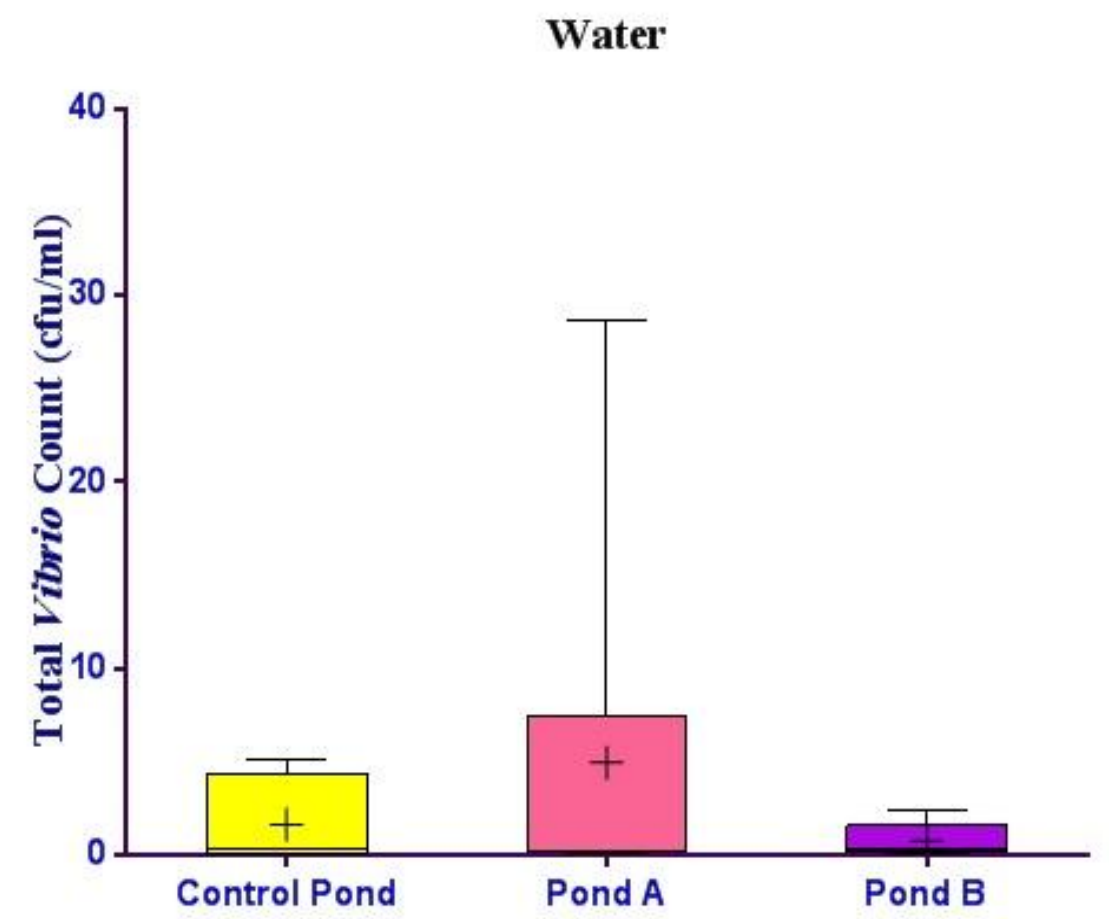

Gut




Fig.5 Total vibrio count of culture ponds at Vadacheepurupalli during summer in the year 2013

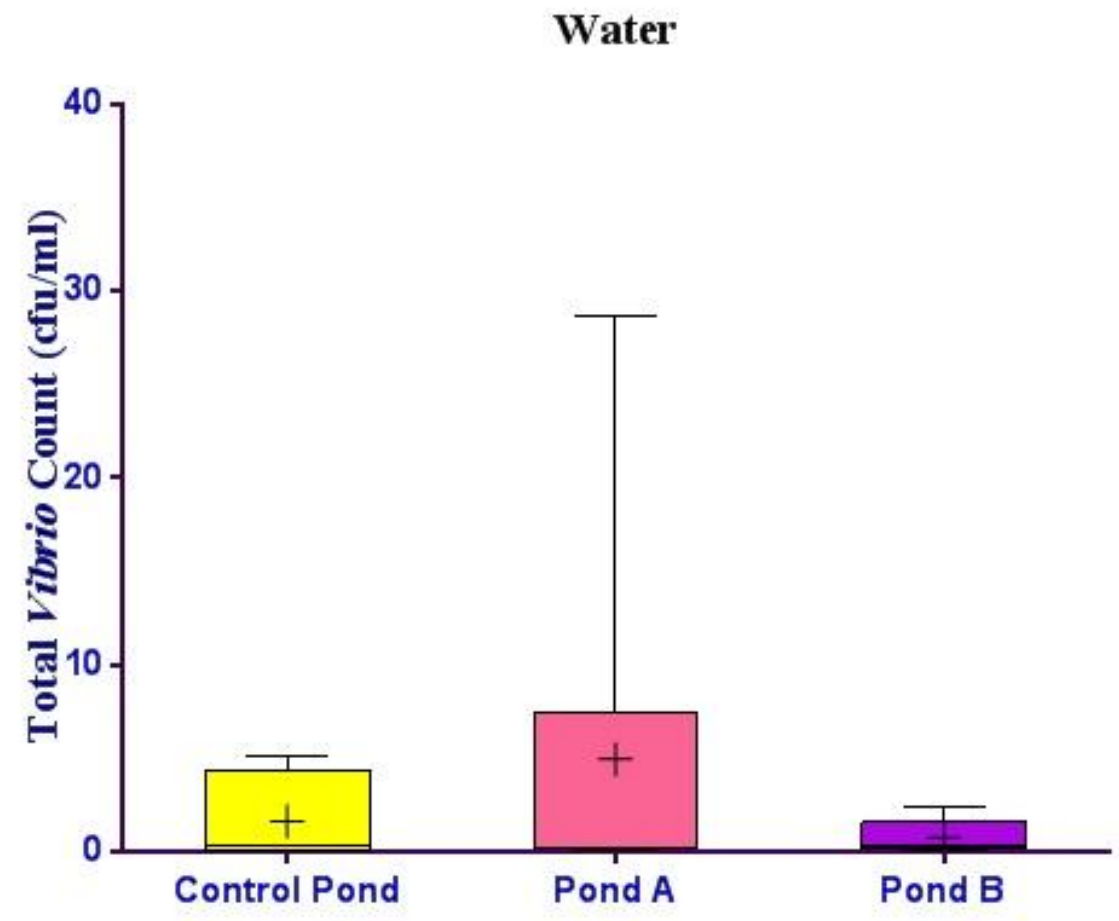

Gut

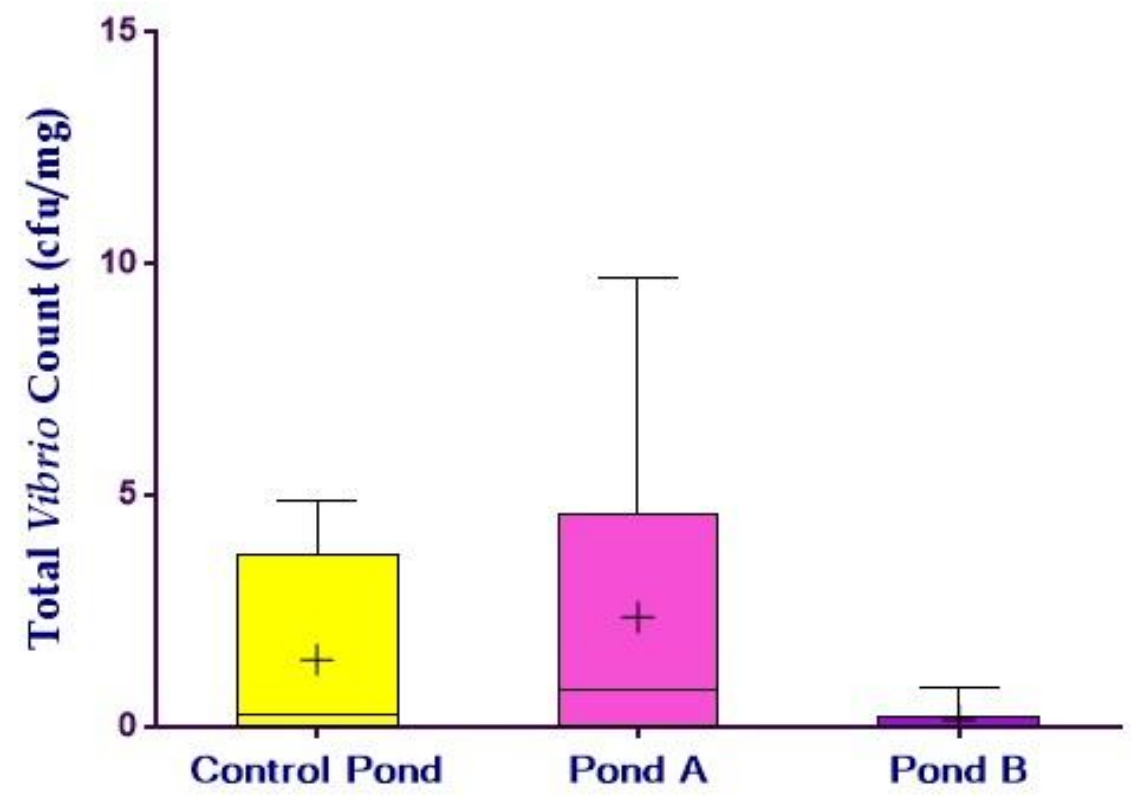


Fig.6 Total vibrio count of culture ponds at Vadacheepurupalli during winter in the year 2013

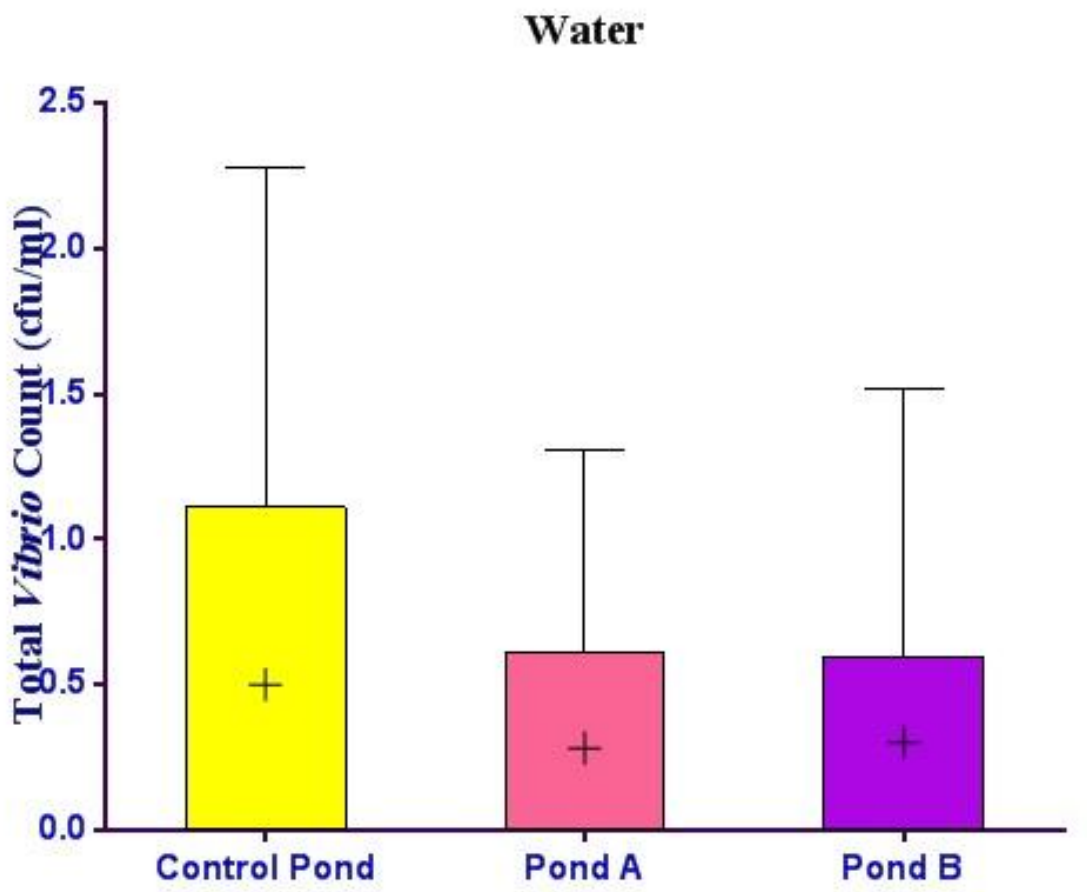

\section{Gut}

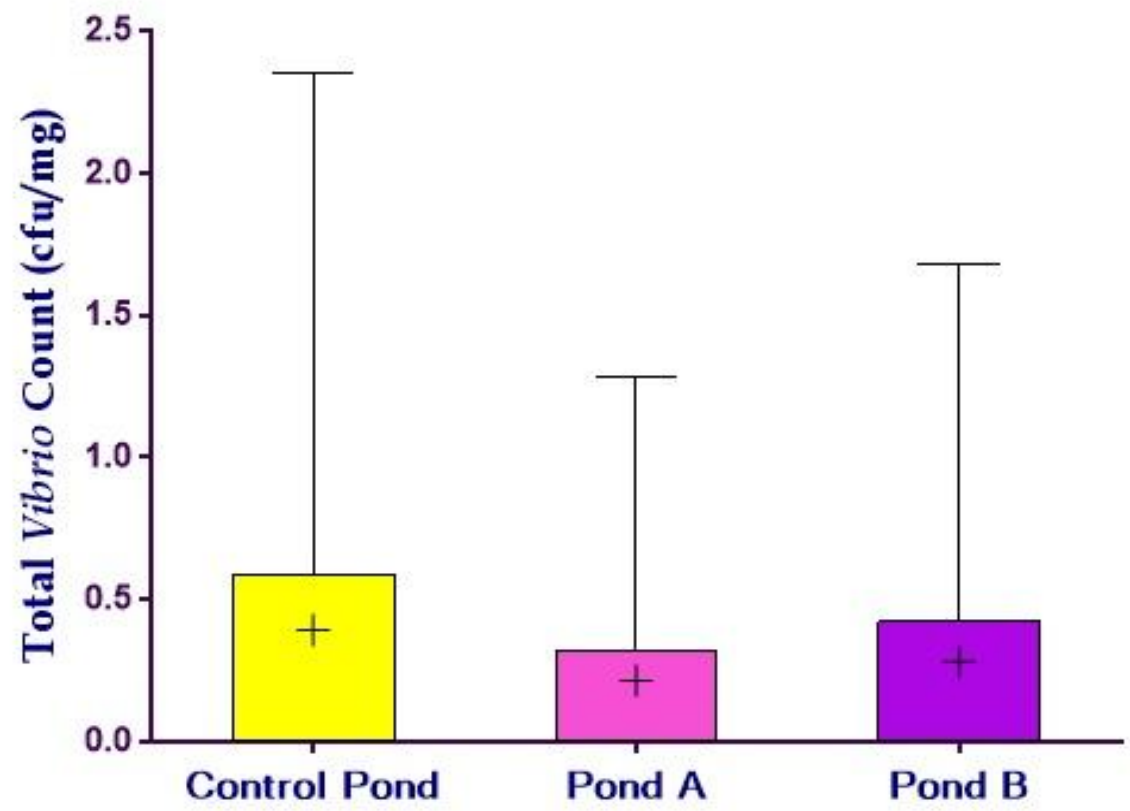

In conclusion, the present study was undertaken to study the effect of organic acids and probiotics in culture ponds of Litopenaeus vannamei over a period of three consecutive years from 2011 to 2013. The effect of Organic acids and probiotics created a healthy 
and suitable environment for the shrimp culture. These have decreased the pathogens thereby decreasing the onset of various diseases. The growth and survival rate have been greatly influenced.

Application of these in the closed recirculating system in a semi intensive culture has been successful and the output has been marginally well over the conventional method. Based on the results of the present study, it was revealed that the application of probiotics have controlled the pathogenic Vibrio spp.in the shrimp culture pond and in the gut by the effect of organic acids. It is evident from the experimental ponds $\mathrm{A} \& \mathrm{~B}$, which showed considerable reduction in the Total vibrio count (TVC) during the culture period

\section{Acknowledgement}

We thank Management of Karuna Aqua farms, Visakhapatnam for the financial support and facilities provided to make this work possible.

\section{References}

da Silva, B.C., Vieira, F.N., Mouriño, J.L.P., Ferreira, G.S., Seiffert,W.Q., 2013. Salts of organic acids selection by multiple characteristics for marine shrimp nutrition. Aquaculture, 384-387: 104110.

Freitag, M. 2007. Organic acids and salts promote performance and health in animal husbandry. In: Acidifiers in Animal Nutrition-A Guide for Feed Preservation and Acidification to Promote Animal Performance (Ed. C. Lückstädt). Nottingham University Press.pp.1-11.

Freitag, M. 2007. Organic acids and salts promote performance and health in animal husbandry. In: Acidifiers in Animal Nutrition-A Guide for Feed Preservation and Acidification to Promote Animal
Performance (Ed. C. Lückstädt). Nottingham University Press.pp.1-11.

Gatesoupe, F.J. 1999. The use of probiotics in aquaculture. Aquaculture, 180: 147-165.

Gomes, R., Geovannay, D., Balcazar Jose Luis., Ma Shen. 2007. Probiotics as control agents in aquaculture. J. Ocean University of China, Volume 6, PP 76-79.

Gómez, R., Geovanny, D., Shen, M.A. 2008. Influence of probiotics on the growth and digestive enzyme activity of white Pacific shrimp (Litopenaeus vannamei). Ocean Coast Sea Res., 7: 215-218.

Gullian, M., Thompson, F., Rodriguez, J. 2004. Selection of probiotic bacteria and study of their immunostimulatory effect in Penaeus vannamei. Aquaculture, 233: 114.

Jin, L.Z., Ho, Y.W., Abdullah, N., Jalaludin, S. 1997. Probiotics in poultry: modes of action. World's Poultry Sci. J., 53(4): 351-368.

Mine, S., Boopathy, R. 2011. Effect of organic acids on shrimp pathogen, Vibrio harveyi. Curr. Microbiol., 63: 1-7.

Mohamed, K.S. 1995. Probiotics - An emerging concept in aquaculture nutrition and disease control, Seafood Export J., 26(7): 5-9.

Moriarty, D.J.W. 1999. Disease control in shrimp aquaculture with probiotic bacteria. Proceedings of the $8^{\text {th }}$ International Symposium on Microbial Ecology, pp 115-120.

Ng, W.K., Koh, C.B. 2011. Application of organic acids in aqua feeds: impacts on fish growth, nutrient utilisation and disease resistance. In: Luckstadt, C. (Ed.), Standards for acidifiers, principles for the Use of organic acids in animal nutrition. Nottingham University Press, Nottingham, United Kingdom, pp. 49-58.

Ng, W.K., Koh, C.B., Sudesh, K., Siti-Zahrah, A. 2009. Effects of dietary organic acids on growth, nutrient digestibility and gut microflora of red hybrid tilapia, Oreochromis sp., and subsequent survival during a challenge test with Streptococcus agalactiae Aquac. Res., 40: 1490-1500. 
Prabhu, N.M., Nazar, A.R., Rajagopal, S., Khan, S.A. 1999. Use of probiotics in water quality management during shrimp culture. J. Aqua Trop., 14: 227-236.

Rahman, M.M., Escobedo-Bonilla, C.M., Corteel, M., Dantas-Lima, J.J., Wille, M., Alday-Sanz, M.V., Pensaert, M.B., Sorgeloos, P., H.J. Nauwynck. 2006. Effect of high water temperature $\left(33^{\circ} \mathrm{C}\right)$ on the clinical and virological outcome of experimental infections with white spot syndrome virus (WSSV) in specific pathogen-free (SPF) Litopenaeus vannamei. Aquaculture, 261: 842-849.

Ravichandran, R., J.R. Shaick., R. Jalaluddin. 2001. Stress management strategy with probiotics for preventing shrimp diseases. Appl. Fisheries and Aquaculture, 1: 7374.

Rebouças, R.H., de Sousa, O.V., Lima, A.S., Vasconcelos, F.R., de Carvalho, P.B., dos Fernandes Vieira, R.H.S. 2011. Antimicrobial resistance profile of Vibrio species isolated from marine shrimp farming environments (Litopenaeus vannamei) at Ceará, Brazil. Environ. Res., 111: 21-24.

Rosas, C., Cuzon, G., Gaxiola, G., LePriol, Y., Pascual, C., Rossignyol, J., Contreras, F., Sánchez, A., Van Wormhoudt, A. 2001. Metabolism and growth of juveniles of Litopenaeus vannamei: effect of salinity and dietary carbohydrate levels. J. Exp. Mar. Biol. Ecol., 2591-22.

Van Hai, N., Buller, N., Fotedar, R. 2009. Effects of probiotics (Pseudomonas synxantha and Pseudomonas aeruginosa) on the growth, survival and immune parameters of juvenile western king prawns (Penaeus latisulcatus Kishinouye, 1896). Aquac. Res., 40: 590-602.

Vaseeharan, B., Ramasam, P. 2003. Control of pathogenic Vibrio spp. by Bacillus subtilis BT23, a possible probiotic treatment for black tiger shrimp Penaeus monodon. Lett. Appl. Microbiol., 36(2): 83-87.

Vaseeharan, B.J., Lin, P. Ramasamy. 2004. Effect of probiotics, antibiotics sensitivity, Pathogenesity and plasmid profiles of Listonella anguillarum - like bacterial isolated from Peneausmonodon culture systems. Aquaculture, volume 241, Issue 1-4, p. 77-91.

Verschuere, L., Rombaul, G., Sorgeloos, P., Verstraete, W. 2000. Probiotic bacteria as control agents in aquaculture, Microbiol, Mol. Biol.Rev., 64: 655-671.

Vine, N.G., Leukes, W.D., Kaiser, H., Daya, S., Baxter, J., Hecht, T. 2004a. Competition for attachment of aquaculture candidate probiotic and pathogenic bacteria on fish intestinal mucus. J. Fish Dis., 27(6): 319326.

Wang, Y., Xu, Z., Xia, M. 2005. The effectiveness of commercial probiotics in Northern White Shrimp Penaeus vannamei ponds. Fisheries Sci., Vol. 71, No. 5, pp. 1034-1039.

Zhou, X., Wang, Y. Li, W. 2009. Effect of probiotic on larvae shrimp (Penaeus vannamei) based on water quality, survival rate and digestive enzyme activities. Aquaculture, 287: 349-353.

\section{How to cite this article:}

Gopal Anand Kandikatla and Ramesh Babu Kondamudi. 2016. Effect of Organic Acids and Probiotics on the Pond Ecosystem in the Culture Ponds of Litopenaeus vannamei. Int.J.Curr.Microbiol.App.Sci. 5(6): 314-330.

doi: http://dx.doi.org/10.20546/ijcmas.2016.506.036 